\title{
A Evolução da Insulinoterapia no Diabetes Melito Tipo 1
}

revisão

\author{
antonio Carlos Pires \\ ANTONIO Roberto Chacra
}

Disciplina de Endocrinologia e

Metabologia da Faculdade de Medicina de São José do Rio

Preto (Famerp), SP, Brasil (ACP);

Disciplina de Endocrinologia da Escola Paulista de

Medicina da Universidade Federal de São Paulo

(EPM/Unifesp) (ARC), SP, Brasil.
RESUMO

A descoberta da insulina foi o grande marco da história do diabetes melito e a grande conquista para o seu tratamento. A primeira insulina disponibilizada foi a regular. Na seqüência, Hagedorn acrescentou a protamina à insulina, criando, assim, a insulina NPH. Na década de 1950 foi sintetizada uma insulina desprovida de protamina, denominada insulina lenta. Com o advento da biologia molecular, sintetizou-se, via DNA recombinante, a insulina humana sintética. Mais recentemente, foram disponibilizados vários tipos de análogos de insulina que permitiram o melhor controle metabólico dos pacientes. O tratamento do diabetes melito tipo 1, além do processo educacional, incluindo a prática regular de atividades físicas e orientações dietéticas, resumese na substituição plena de insulina de longa e curta durações de ação, de maneira individualizada, de acordo com a experiência do médico-assistente. No diabetes melito tipo 1, a preferência é pelas insulinas de menor variabilidade, por meio do esquema basal/bólus ou pelas bombas de infusão contínua de insulina subcutânea com o objetivo de mimetizar a liberação fisiológica de insulina pelas células- $\beta$. (Arq Bras Endocrinol Metab 2008;52/2:268-278)

Descritores: Insulinas; História; Tratamento; Diabetes melito tipo 1

\section{ABSTRACT}

Insulin Therapy for Type 1 Diabetes Mellitus: Past and Present.

The discovery of insulin can be considered the milestone of diabetes mellitus history and a great achievement for its treatment. The first insulin available was the regular. Afterwards, Hagedorn added the protamine to the insulin, thus, creating the NPH insulin. In the 1950s an insulin free of protamine was synthesized: the lente insulin. With the advent of molecular biology, synthetic human insulin was synthesized using recombinant DNA technology. Most recently several types of insulin analogues were available, providing the patients with better metabolic control. Type 1 diabetes mellitus treatment includes plain substitution and individualization for short-acting plus long-acting insulin according to the physician's assistance, besides regular practice of physical activities and diet orientations. In type 1 diabetes mellitus the insulin of low variability is the best choice since basal/bolus insulin therapy or continuous subcutaneous insulin infusion pump can mimetize the physiological release of insulin by $\beta$ cells. (Arq Bras Endocrinol Metab 2008;52/2:268/278)

Keywords: History; insulin; Type 1 diabetes mellitus; Therapy 


\section{HISTÓRIA DA INSULINA}

DIABETES MELITO É UMA doença tão antiga quanto a própria humanidade. O papiro de Ebers, manuscrito da época 1500 a.C., menciona esta entidade e chama a atenção para a diurese freqüente e abundante, sede incontrolável e emagrecimento acentuado, como suas principais manifestações clínicas. Aretaeus, médico romano, criou o termo dia-betes que significa "passar através" por causa de excessiva diurese, um dos sintomas mais evidentes da doença, ser parecido à drenagem de água por meio de um sifão.

No século VI, médicos hindus descreveram mais detalhadamente alguns sintomas da doença e relataram, pela primeira vez, o sabor adocicado da urina destes indivíduos. Em 1869, Langerhans identificou conjuntos de células no tecido pancreático que denominou ilhotas celulares. Em 1889, na França, Joseph von Mering e Oskar Minkowski da Universidade de Strasbourg, durante pesquisas sobre a digestão de gorduras, observaram que a remoção do pâncreas de cães desencadeava sinais e sintomas similares aos do diabetes. Em 1908, o cientista alemão Georg Zuelzer desenvolveu o primeiro extrato pancreático injetável que suprimiu a glicosúria. Infelizmente, esta modalidade terapêutica não evoluiu em virtude de importantes efeitos adversos (1). Em 14 de novembro de 1899, nascia em Ontário, Frederick Banting que, em 1912, iniciou o curso de graduação em Medicina na Universidade de Toronto.

Entre 1910 e 1920 emerge nos Estados Unidos um dos mais importantes diabetologistas da época, Elliot P. Joslin, que definia diabetes como uma doença crônica, não-contagiosa, que evoluía sem dor e passível de ser tratada cronicamente.

Em 1921, Banting e Charles Best, no laboratório do fisiologista JJR MacLeod, durante estudos em cães tentando demonstrar que a secreção exócrina pancreática poderia destruir o composto químico sintetizado pelas ilhotas de Langerhans, descobriram e isolaram a insulina. A descoberta da insulina foi o grande marco da história do diabetes melito e a grande conquista para o tratamento e a sobrevida dos pacientes. Em 11 de janeiro de 1922, clínicos do Toronto General Hospital prescreveram de modo injetável $15 \mathrm{ml}$ de extrato pancreático a um paciente com diabetes, Leonard Thompson de 14 anos de idade em estado clínico crítico. Houve poucos efeitos sobre a glicosúria e a cetonúria e o pior, evoluiu com formação de abscesso estéril no local da aplicação. Diante deste fato, o bioquímico JB Collip purificou este extrato pancreático e em seguida foi novamente aplicado ao mesmo paciente, desta vez com resposta imediata e eficaz da glicosúria e da cetonúria. Com estes achados, pela primeira vez na história ficou demonstrado, de maneira inequívoca, a relação da secreção interna pancreática e o diabetes melito. Em razão destas conquistas terapêuticas, em 1923, Banting e Macleod receberam do Nobel Committee of the Caroline Institute, o prêmio Nobel de Medicina e Fisiologia (2-4).

Para a prática clínica, o acontecimento mais importante após estes achados experimentais, foi o acordo com a Eli Lilly and Company of Indiana para a industrialização e a comercialização em larga escala da insulina. A primeira insulina a ser comercializada foi denominada insulina regular ou insulina " $R$ ". Por causa de seu efeito clínico de curta duração, exigia três ou quatro aplicações diárias para o bom controle metabólico. Sobretudo, a introdução da insulina rápida na terapêutica do diabetes mudou de maneira drástica a expectativa de vida dos indivíduos recém-diagnosticados; uma vez que a fase pré-insulina que era de 2,6 anos, passou para 6,0 anos em média, melhorando progressivamente com a evolução global da terapêutica (5). Um dos primeiros europeus a visitar Toronto para conhecer mais detalhadamente os experimentos com a insulina foi o dinamarquês August Krog junto com HC Hagedorn, pelo fato de sua esposa ser portadora de diabetes melito. Após o período experimental, iniciaram a produção comercial de insulinas na indústria farmacêutica denominada Nordisk Laboratory, em Copenhague.

Em 1926, John Jacob Abel, do Johns Hopkins Hospital (Baltimore) cristalizou a insulina, que finalmente foi reconhecida como hormônio protéico (6). As primeiras preparações de insulinas foram extraídas de animais. Em virtude de formulações de ações rápidas houve na época muitas queixas de pacientes e familiares por causa da necessidade de uso de múltiplas injeções diárias. A partir daí, desencadeou-se a preocupação e o interesse por parte das indústrias farmacêuticas em prolongar o tempo de ação das insulinas.

Entre 1930 e 1940, Hagedorn na Dinamarca acres centou à insulina uma proteína básica denominada protamina, desenvolvendo, assim, a insulina NPH. Simultaneamente, Scott e Fisher em Toronto, com a adição de zinco à molécula de insulina, sintetizaram a Protamine-Zinc Insulin (PZI). 
No início da década de 1950, na Dinamarca foi lançada a insulina lenta, desprovida de protamina. Nos 20 anos seguintes, as insulinas PZI, NPH e lenta supriram o mercado mundial para o tratamento de diabetes melito. Contudo, na prática clínica, diferentes complicações do uso destas insulinas foram observadas, entre elas, quadros alérgicos, lipodistrofias nos locais das aplicações e, a mais importante, resistência imunológica à insulina.

Em 1973, visto como grande evolução tecnológica da época foi desenvolvida e lançada no mercado uma nova preparação de insulina porcina livre de peptídios imunogênicos, denominada insulina monocomponente (7).

Com o advento da biologia molecular, via DNA recombinante, iniciou-se a era das insulinas biossintéticas humanas, utilizadas por muitos pacientes até os dias atuais. Por meio de injeções subcutâneas em comparação com a insulina animal, a insulina humana sintética apresenta farmacodinâmica e farmacocinética diferentes. A insulina humana tende a ser de absorção mais rápida e de período de ação mais curto, mas com picos de ações que ocorrem de maneira totalmente imprevisíveis. É importante citar que na prática diária, estas diferenças não são tão significantes quando a insulina humana é usada com estratégias terapêuticas adequadas (8).

No final da década de 1990, a indústria farmacêutica Eli Lilly Company sintetizou a insulina de ação ultra-rápida denominada lispro, que quimicamente se fundamentou na inversão de posições dos aminoácidos prolina (B28) e lisina (B29) na cadeia B da insulina humana, tornando-a similar à estrutura química do Insulin-like Growth Factor I (IGF-1). A importância desta inversão é que pôde acelerar a absorção por causa de formação de hexâmeros que se dissociam rapidamente. Em seguida, foi introduzida no mercado a insulina asparte que quimicamente se diferencia da insulina humana pela substituição do aminoácido prolina na posição B28 da cadeia B da insulina pelo ácido aspártico. Estes análogos de ação ultra-rápida apresentam perfis similares, tanto da farmacocinética como da farmacodinâmica. Na época do lançamento, estes análogos trouxeram grandes expectativas para os diabetologistas e clínicos pelo potencial redutor de riscos de episódios de hipoglicemias, principalmente os eventos noturnos. Comparando com estas formulações ultra-rápidas, a insulina regular, pelo fato de conter zinco em sua formulação, precipita facilmente no subcutâneo em hexâmeros de dissociação mais lenta proporcionando, assim, absorção mais tardia do que as insulinas lispro e asparte $(9,10)$.

Em 2000, outro análogo de insulina, desta vez de ação prolongada, denominado de glargina, foi aprovado pela Food and Drugs Administration (FDA) e European Medicines Evaluation Agency (EMEA) para o uso em pacientes com diabetes tipos 1 e 2 . A estrutura química da glargina difere da insulina humana em três posições de aminoácidos. Na cadeia A21, a asparagina é substituída pela glicina para aumentar a estabilidade da molécula e duas moléculas de argininas são acrescentadas na posição B31 e B32. Estas alterações mudam o ponto isoelétrico da insulina, elevando o seu $\mathrm{pH}$ para o mais próximo possível do neutro. Apesar disso, o $\mathrm{pH}$ levemente ácido promove no tecido subcutâneo a formação de micropreciptados, lentificando, assim, sua absorção para a circulação sanguínea. Além disso, para otimizar a estabilização da molécula são adicionadas pequenas quantidades de zinco que contribuem ainda mais para a lentificação de sua absorção pelos capilares sanguíneos. Em vários ensaios clínicos envolvendo pacientes com diabetes tipos 1 ou 2, comparando a glargina com a insulina NPH, a glargina demonstrou início de ação mais lento, com efeito mais prolongado, estável e picos pouco pronunciados $(11,12)$.

Recentemente, mais um análogo de insulina de ação prolongada, denominado detemir foi aprovado pelas agências regulatórias, FDA e EMEA. A insulina detemir é um composto solúvel em pH neutro e basicamente foi desenvolvida com o objetivo de obter valores glicêmicos mais estáveis e previsíveis. Foi sintetizada a partir da acilação do ácido mirístico na posição B29 da insulina humana, onde está posicionada a lisina, e também a remoção do aminoácido treonina da posição B30. O ácido mirístico é um ácido graxo de 14 carbonos com a função de se ligar à albumina de modo reversível, tanto no interstício como no plasma. Portanto, este processo de "liga e desliga" da albumina proporciona à insulina detemir uma ação previsível e prolongada (13-15) (Figura 1).

Aprovada em 2006 pela FDA e em seguida disponibilizada ao mercado, a insulina humana inalável de ação rápida é indicada para o uso pré-prandial em pacientes com diabetes melito tipo 1 ou 2. Do ponto de vista farmacológico, tem pico de ação similar a dos análogos de efeito rápido e duração de atividade hipoglicemiante comparável à da insulina humana regular em uso subcutâneo (16-18). 


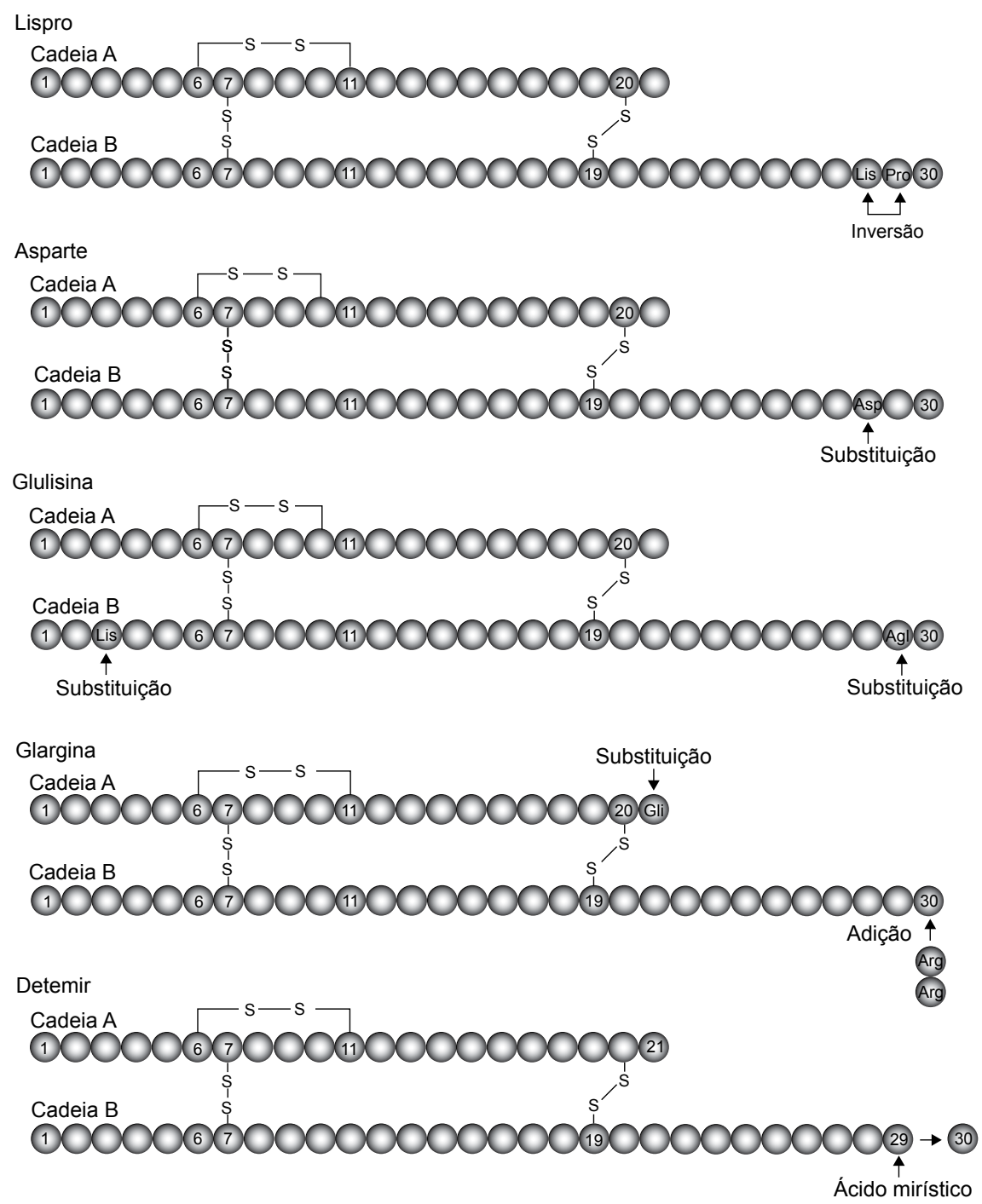

Figura 1. Mudanças nas seqüências dos aminoácidos dos análogos lispro, asparte, glulisina, glargina e detemir (adaptadas de Hirsch; Pawlic-Renar e cols. (9-13).

Em resumo, nestes últimos 85 anos, desde a descoberta da insulina, houve importantes avanços na insulinoterapia. Entre eles, os mais importantes foram a purificação da insulina animal, a substituição pela insulina humana sintética e, mais recentemente, a síntese de análogos de insulina de ação rápida e prolongada. Finalmente, outra importante conquista para a insulinoterapia foi o lançamento da formulação em pó da insulina humana, utilizada de maneira inalada e absorvida pela circulação pulmonar. Todos estes avanços ao longo do tempo tiveram como objetivo proporcionar maiores facilidades ao paciente e, obviamente, melhores resultados no controle metabólico.

\section{Insulinoterapia no diabetes melito tipo 1}

De acordo com Eisenbarth (19), o diabetes melito do tipo 1 pode ser definido como uma doença auto-imune geneticamente programada. Didaticamente, sua patogenia é subdividida em estágios evolutivos desde a suscetibilidade genética, passando pela ativação do sistema imunológico desencadeada por fatores precipitantes ambientais até a falência total ou quase total das células- $\beta$. É classificado pela American Diabetes Association Expert Committee em tipo IA como auto-imune ou imunemediado e o tipo $1 \mathrm{~B}$ não auto-imune ou idiopático (20). O manejo terapêutico e as modalidades de prevenção de suas complicações têm evoluído de maneira bastante evi- 
dente nos últimos anos. $\mathrm{O}$ tratamento intensivo com a reposição insulínica, maiores cuidados com a orientação alimentar e educacional para os pacientes e seus familiares e a preocupação com outros fatores de risco vasculares são de fundamental importância no acompanhamento destes indivíduos (21-23).

A insulinoterapia no diabetes melito tipo 1 começou com a insulina regular com múltiplas aplicações diárias. Após o advento das insulinas de ações intermediárias ou de ações mais prolongadas, os pacientes passaram a usar apenas uma ou duas aplicações diárias. Em um interessante estudo, publicado na década de 1960, avaliando a incidência de retinopatia após 15 anos de diagnóstico de diabetes, comparou-se dois grupos de pacientes tratados antes e após 1935. Os indivíduos tratados com múltiplas injeções diárias de insulina mostraram incidência significativamente menor de retinopatia do que aqueles tratados com somente uma ou eventualmente duas aplicações diárias (24). Não há evidências com estudos randomizados comparando-se resultados de longo prazo de insulinas tradicionais com os novos regimes terapêuticos envolvendo os análogos de insulina.

Os análogos de curta duração têm propriedades químicas diferentes da insulina regular, mas suas vantagens terapêuticas no controle da glicemia em crianças e adolescentes ainda estão sob debate $(25,26)$. Ao mesmo tempo, em relação aos riscos de eventos de hipoglicemias, os análogos de insulina de ação rápida mostram sem dúvida, melhores e significantes resultados $(27,28)$. Em relação à insulinização basal, os análogos glargina e detemir demonstram resultados superiores e significantes, com menor variabilidade diária das glicemias quando comparados com a insulina humana NPH.

Com o preceito de que o tratamento rigoroso da glicemia em diabetes melito tipo 1 reduz os riscos de complicações agudas e crônicas e ao mesmo tempo diante do desafio de se conseguir bons resultados terapêuticos em adolescentes, Dunger e cols. (29) avaliaram o controle glicêmico e a incidência de episódios hipoglicêmicos noturnos em adolescentes em regime de uma injeção diária de glargina combinada a múltiplas aplicações diárias de lispro com NPH em duas aplicações diárias mais insulina regular às refeições. Os resultados demonstraram que as glicemias nos períodos pré e pós-refeições, exceto do jantar, foram inferiores e significantes com o uso dos análogos. Os valores das hemoglobinas glicadas foram similares nos dois grupos. Entretanto, a incidência de episódios hipoglicêmicos noturnos foi $43 \%$ menor no grupo com os análogos. Na prática, a maior previsibili- dade destes análogos é traduzida pelo menor risco de episódios graves de hipoglicemias, principalmente no período noturno $(14,30)$.

Vários estudos randomizados demonstram melhores resultados no controle glicêmico com múltiplas aplicações diárias de insulina ou com a infusão contínua subcutânea de insulina quando comparados com duas aplicações diárias de insulina humana $(31,32)$. No momento, a infusão contínua subcutânea de insulina é o meio mais adequado para imitar a liberação fisiológica de insulina pelo pâncreas. Hirsch e cols. compararam dois esquemas de reposição insulínica, um com múltiplas doses diárias em bólus de insulina asparte e basal com glargina versus a infusão contínua subcutânea de insulina asparte em pacientes com diabetes melito tipo 1. Avaliaram os resultados fundamentados nos valores de frutosamina, que foram significativamente inferiores no grupo em uso de infusão, quando comparado com o regime de múltiplas aplicações diárias de insulina. A incidência de episódios hipoglicêmicos foi similar em ambos os grupos. Contudo, é importante salientar que uma das mais importantes barreiras para a implementação deste meio terapêutico é o seu custo mais elevado comparado aos métodos convencionais de insulinização (33).

Com base em estudos prévios envolvendo crianças em idade escolar e adolescentes, Alemzadeh e cols. (34) avaliaram durante dois anos consecutivos dois esquemas de insulinoterapia em um mesmo grupo de crianças na faixa etária entre 2,6 e 6,3 anos, com média de 4,8 \pm 1,0. No primeiro ano, utilizou-se o regime denominado múltiplas doses diárias (MDD) que constituiu de uma dose diária de glargina ao deitar-se associada à insulina lispro em horários de refeições preestabelecidos. No segundo ano, trocou-se o esquema para o denominado múltiplas doses diárias em horários flexíveis (MDDF), ou seja, manteve-se a glargina ao deitar-se, mas a lispro foi utilizada de acordo com o horário de alimentação de escolha das crianças ou dos adolescentes. Nos indivíduos com peso normal, houve queda mais acentuada e significante da hemoglobina glicada com redução no número de eventos hipoglicêmicos no período em uso do esquema MDDF. É importante observar que há muito tempo, a hipoglicemia grave em crianças com idade abaixo de 6 anos de idade tem sido uma preocupação do médico e da família por causa dos riscos de seqüelas neurocognitivas irreversíveis (35-37). Embora, o análogo de insulina glargina não tenha sido aprovado para uso em crianças com diabetes abaixo dos 6 anos de idade pelo FDA, Dixon e cols. (38) com o objetivo de avaliar o uso de glargina nesta 
população especial, compararam dois períodos de tratamento. Um período, seis meses antes em uso de insulina $\mathrm{NPH}$ e o outro seis meses depois em uso de glargina. Em relação aos eventos hipoglicêmicos graves houve significante redução de 16 para três episódios/período com o uso de glargina. Quanto à hemoglobina glicada, não houve diferença significante entre os períodos analisados.

Os ensaios clínicos, The Diabetes Control and Complications Trial (DCCT) e Epidemiology of Diabetes Interventions and Complications (EDIC) demonstraram melhor controle metabólico e redução de risco de complicações microvasculares. Ainda, menor risco de eventos cardiovasculares ao longo do acompanhamento dos pacientes submetidos ao tratamento intensivo com múltiplas aplicações diárias ou com a infusão contínua subcutânea de insulina quando comparados ao tratamento convencional com duas aplicações diárias de insulina humana $(39,40)$. De qualquer maneira, no diabetes melito tipo 1 , a insulinoterapia deve ser iniciada o mais rapidamente possível, no máximo até seis horas após o diagnóstico, para prevenir a cetoacidose ou outras complicações agudas (28).

\section{TIPOS DE INSULINAS DISPONÍVEIS}

\section{Regular}

A insulina humana solúvel com início de ação entre 30 e 60 minutos, com pico de ação entre 2 e 4 horas e término entre 6 e 10 horas quando usada por via subcutânea, normalmente, é indicada no tratamento da cetoacidose diabética e também associada com insulina humana de ação intermediária ou com análogos basais no período de 20 a 30 minutos pré-refeições, com o objetivo de reduzir o pico de glicemia pós-prandial.

\section{Análogos de insulina de ação rápida}

A lispro e a asparte, análogos de insulina de ação rápida, estão disponíveis para o uso clínico e mostram propriedades farmacocinética e farmacodinâmica similares. A lispro em sua preparação farmacêutica com fenol e zinco forma hexâmeros estáveis que se dissociam rapidamente em monômeros no tecido subcutâneo. A insulina regular em comparação a estes análogos apresenta dissociação em monômeros de maneira mais lenta. Em uso subcutâneo, ambos têm início entre 5 e 15 minutos com pico entre 1 e 2 horas e término de ação entre 4 a 6 horas. São opções indicadas para as bombas de infusão contínua subcutânea de insulina, imediatamente antes das refeições em esquema basal/bólus e também, em picos hiperglicêmicos esporádicos ao longo do dia.
Não raro, em uso destes análogos há necessidade de dose adicional no período da tarde para compensar a hiperglicemia resultante do lanche vespertino. Em comparação à insulina regular, existem evidências que, além de reduzir picos hiperglicêmicos pós-prandiais, reduzem também o risco de hipoglicemias, principalmente no período noturno $(9,41-44)$. Um novo análogo de ação rápida ainda não disponível no mercado brasileiro é a glulisina. É sintetizada a partir da insulina humana com duas mudanças na seqüência dos aminoácidos da cadeia B. Na posição B3, a asparagina é substituída pela lisina e na posição B29, a lisina é substituída pelo ácido glutâmico. Este análogo tem propriedades farmacodinâmicas e farmacocinéticas similares às insulinas lispro $\mathrm{e}$ asparte (45-49).

\section{Insulinas de ação intermediária}

As preparações de insulina lenta e NPH representam esta categoria. Elas apresentam início de ação entre $\mathrm{l}$ e 2 horas com pico entre 4 e 8 horas e término entre 12 e 20 horas. São usadas em regimes basais em duas ou mais aplicações diárias. Podem ser misturadas à insulina regular na mesma seringa para facilitar a adesão da insulinoterapia, principalmente em crianças na fase pré-escolar. É importante ressaltar que pelo excesso de zinco na insulina lenta este procedimento pode prolongar o efeito da insulina regular $(28,50)$.

\section{Análogos de insulina basal}

As formulações glargina e detemir representam os grupos denominados análogos de longa duração ou basais. A farmacodinâmica e a farmacocinética são mais previsíveis e os picos são pouco pronunciados quando comparadas com as insulinas NPH e lenta. Apresentam início de ação entre 1 e 2 horas, atingem o platô de ação biológica entre 4 e 6 horas com término de efeito entre 20 e 24 horas. A glargina pelo seu pH levemente ácido não pode ser misturada a outras insulinas na mesma seringa. Em indivíduos portadores de diabetes melito tipo $1, \mathrm{o}$ análogo glargina pode ser aplicado antes do café da manhã, antes do jantar ou antes de dormir, embora os episódios de hipoglicemias noturnos parecem ser menos freqüentes quando é administrado pela manhã. Por outro lado, em virtude de seu $\mathrm{pH}$ ácido algumas crianças podem se queixar de sensação de queimação no local da aplicação.

Em um interessante estudo randomizado duplocego, por meio de clampe euglicêmico, em 12 pacientes com diabetes melito do tipo 1 avaliou-se a duração 
de ação da insulina detemir com diferentes e crescentes doses de 0,$1 ; 0,2 ; 0,4 ; 0,8$ e $1,6 \mathrm{IU} / \mathrm{kg}$ comparadas com uma dose de $0,3 \mathrm{IU} / \mathrm{kg}$ de insulina NPH. Os resultados demonstraram que com doses maiores o tempo de ação da insulina detemir foi mais prolongado e, além disso, de maneira inexplicada, mostrou tendência a apresentar picos de ação conforme o aumento das doses (5l). Em pacientes pediátricos, em uso de detemir, a maioria exige duas aplicações diárias para o melhor controle metabólico (14).

Vários ensaios clínicos envolvendo pacientes adultos demonstraram que a detemir tem uma vantagem adicional que é causar menor ganho de peso do que a insulina NPH (52-54). Hermansen e cols. (55) estudaram a tolerabilidade e a eficácia de dois regimes terapêuticos NPH/ regular versus detemir/asparte em indivíduos com diabetes tipo 1 por período de 18 semanas. O controle metabólico foi mais adequado no grupo detemir/asparte, analisado pelo menor valor da hemoglobina glicada, com menor número de episódios hipoglicêmicos e ainda menor incremento de peso corporal $(\mathrm{p}<0,001)$.

\section{Pré-misturas}

Estas preparações estão disponíveis para o uso em diabetes tipo 1 ou tipo 2. A insulina lispro quando cristalizada com protamina resulta uma formulação de ação prolongada denominada Neutral Protamine Lispro(NPL). Esta formulação, quando misturada com lispro nãocristalizada, resulta um composto de $25 \%$ lispro com $75 \%$ NPL denominado no mercado Humalog MIX $75 / 25$. Outro análogo disponível é a associação de $30 \%$ de asparte solúvel com $70 \%$ de asparte ligado à protamina resultando um produto conhecido comercialmente como insulina NovoMix 30. Estes análogos, pelas suas características químicas, limitam a flexibilidade de ajuste individual de doses, principalmente nos pacientes com diabetes melito tipo 1 . Em adolescentes, há evidências mostrando pior controle metabólico quando tratados com estas preparações (56).

\section{Insulina inalável}

É mais uma alternativa eficaz e segura para a insulinoterapia em adultos (57) com diabetes tipo 1 ou tipo 2. É a própria insulina humana, disponível na forma de pó para o uso de maneira inalável. Está disponibilizada em blisters de 1 e $3 \mathrm{mg}$. Cada blister de $1 \mathrm{mg}$ equivale a aproximadamente $3 \mathrm{U}$ e os de $3 \mathrm{mg}$ corresponde a $8 \mathrm{U}$ de insulina humana regular quando usada por via subcutânea. Quando comparada com a insulina regular em regime subcutâneo, a insulina inalável tem absorção e pico de ação mais rápida comparáveis aos análogos de ação rápida. Em um ensaio clínico de fase III, Quattrin e cols. (58) avaliaram a eficácia e a tolerabilidade da insulina inalada em dois grupos de indivíduos com diabetes melito tipo 1 com esquemas terapêuticos diferentes. Um grupo randomizado para insulina inalada 10 minutos antes das refeições mais insulina ultralenta ao deitar-se e o outro grupo, com duas a três injeções de insulina humana regular mais insulina NPH de manhã e ao deitar-se por período de 24 semanas. A redução da $\mathrm{HbAlc}$ foi similar em ambos os grupos, com menor número de episódios hipoglicêmicos no grupo com insulina inalada. Não houve alterações significantes da função pulmonar durante o estudo (Figura 2 e Tabela 1). Entretanto, em outubro de 2007, a única preparação de insulina inalável $\left(\right.$ Exubera $\left.^{\circledR}\right)$ que se tinha no mercado nacional foi retirada, pelo seu fornecedor, até onde se sabe, por motivos econômicos.

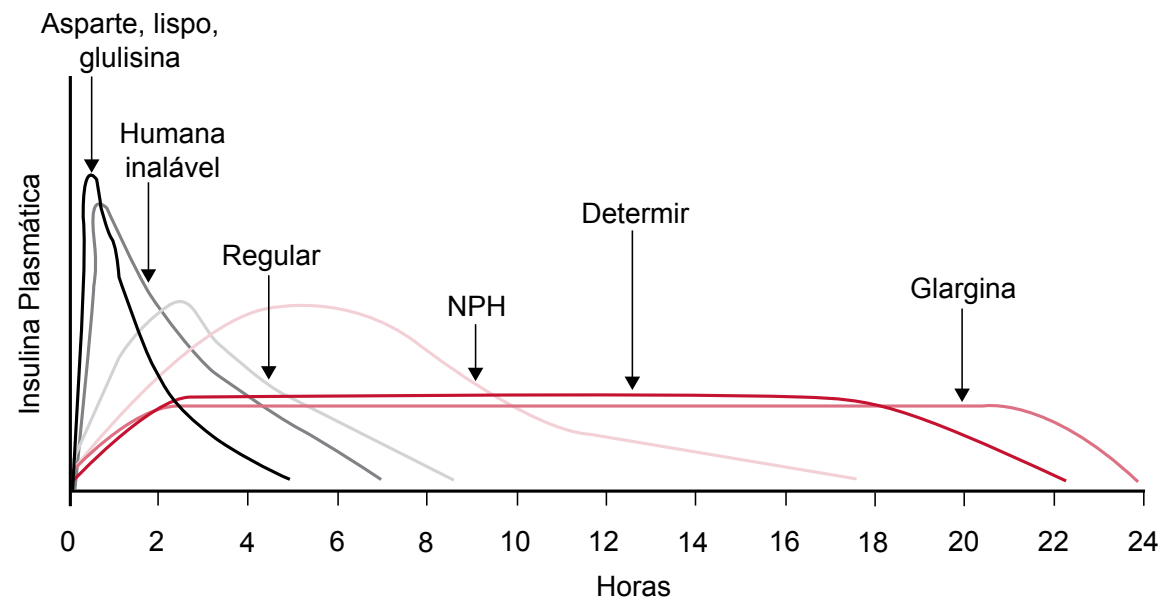

Figura 2. Representação aproximada das farmacocinéticas das insulinas humanas e análogos (adaptada de Hirsch (9)). 
Tabela 1. Início e duração de ação aproximados das insulinas humanas e análogos.

\begin{tabular}{lccc}
\hline Preparação de insulina & Início de ação & Pico & Duração da ação \\
Regular & $30-60$ minutos & $2-4$ horas & $6-10$ horas \\
Humana inalável & $5-15$ minutos & $1-2$ horas & $6-8$ horas \\
NPH/lenta & $1-2$ horas & $4-8$ horas & $10-20$ horas \\
Lispro/asparte/glulisina & $5-15$ minutos & $1-2$ horas & $4-6$ horas \\
Glargina/detemir & $1-2$ horas & pouco pronunciado & $\sim 24$ horas \\
\hline
\end{tabular}

\section{Princípios da insulinoterapia}

Com base nos resultados do DCCT que demonstraram que o controle glicêmico próximo do normal protege contra o início e a progressão das complicações microangiopáticas, o ideal na reposição insulínica é proporcionar, da melhor maneira possível, uma cobertura basal ao longo das 24 horas. O objetivo, assim, seria reduzir a produção hepática de glicose e concomitante, a combinação de bólus de insulina regular ou análogos de ação ultra-rápida pré-refeições para prevenir picos hiperglicêmicos pós-prandiais. É importante salientar que, por causa de riscos de hipoglicemias assintomáticas, principalmente noturnos, nenhuma estratégia de reposição diária de insulina pode otimizar os resultados sem a monitorização glicêmica apropriada. Este procedimento pode ser implementado por meio da automonitorização ou periodicamente com o sistema de monitorização contínua de glicose (MCG) por determinado período. Este método, MCG, é de grande valia, pois permite identificar as flutuações glicêmicas de maneira mais detalhada e, obviamente, propicia subsídios para as mudanças necessárias na orientação terapêutica $(28,59-62)$.

\section{Algumas Sugestões de Esquemas para Ser Utilizados na Insulinoterapia}

a. duas aplicações diárias de insulina NPH, de manhã e ao deitar-se combinadas com insulina humana regular 20 a 30 minutos antes das refeições ou, ainda, análogos de ação ultra-rápidas imediatamente antes das refeições;

b. uma aplicação diária de glargina ou uma a duas aplicações de detemir combinadas com insulinas rápidas ou ultra-rápidas antes das refeições;

c. três a quatro aplicações diárias de insulinas em prémisturas ou bifásicas; d. três a quatro aplicações de insulinas regular ou ultrarápida antes das principais refeições combinadas com insulina NHP, detemir ou glargina ao deitar-se;

e. três a quatro aplicações diárias de insulina NPH combinadas a insulina regular ou ultra-rápidas antes das refeições e ao deitar-se;

f. insulina inalável antes das refeições, combinadas com insulina basal NPH, detemir ou glargina.

Em resumo, é importante enfatizar que a melhor estratégia de reposição de insulina está embasada na preferência e na experiência do clínico ou do especialista, no estado evolutivo da reserva funcional das células- $\beta$, na adesão dos pacientes às orientações educacionais e, fundamentalmente, na análise crítica dos resultados do controle metabólico obtidos por meio da monitorização e dos riscos de complicações micro e macrovasculares ao longo do seguimento $(28,45$, 63-67).

\section{Sugestões de Dosagens Diárias de Insulina}

As dosagens diárias de insulina dependem de muitas variáveis, entre elas as mais importantes ou as mais comuns são a idade, o peso, a duração da doença, o comportamento nutricional e psicológico, as doenças intercorrentes clínicas ou cirúrgicas e os estágios da puberdade $(53,68-70)$.

A seguir, são descritas, independentemente do tipo de insulina prescrita, algumas sugestões práticas para o ajuste de dose total diária. $\mathrm{Na}$ fase de remissão parcial ou "lua-de-mel", < 0,5 IU $/ \mathrm{kg}$, no período pré-púbere entre 0,7 e $1,0 \mathrm{IU} / \mathrm{kg}$ e na puberdade ou nas intercorrências clínicas ou cirúrgicas entre 1 e $2 \mathrm{IU} / \mathrm{kg}$. É evidente que, na prática clínica, a ocorrência de hipo ou hiperglicemias freqüentes e as estratégias terapêuticas de reposição de insulina devem ser cuidadosamente reavaliadas. Freqüentemente, na presença de doenças intercorrentes, 
principalmente infecciosas, ou ainda mudança rápida na rotina de vida do paciente, torna-se necessária a insulinização intensiva com esquema basal/bólus.

Por fim, é importante afirmar que a melhor dose de insulina é aquela ajustada individualmente; é a que promove controle metabólico o mais apropriado possível sem desencadear episódios freqüentes de hipoglicemias, principalmente noturnos; e a que, ao mesmo tempo, proporciona crescimento adequado aos indivíduos (28).

\section{CONSIDERAÇÕES FINAIS}

A incidência de diabetes melito tipo 1 está aumentando rapidamente, principalmente em indivíduos mais jovens. A interface entre suscetibilidade genética e fatores ambientais exerce papel importante na sua patogênese e até o momento, nenhum método tem se mostrado eficaz e seguro na sua prevenção (7l). Na população pediátrica, dados do Western European DM Centers revelam aumento anual da incidência de diabetes tipo 1 entre $3 \%$ e $4 \%$. Sobretudo, este aumento foi mais observado no grupo etário abaixo dos 4 anos de idade, que mostrou taxa anual de $6,3 \%$. Nas faixas etárias entre $5 \mathrm{e}$ 9 e 10 e 14 anos, as taxas de incidência foram de 3,1\% e $2,4 \%$ ao ano, respectivamente (72).

O tratamento do diabetes melito tipo 1 , além do processo educacional, incluindo a prática regular de atividades físicas e orientações dietéticas, resume-se na substituição plena com insulina de longa e curta ação de maneira individualizada, de acordo com a experiência do médico-assistente. As metas são o bom controle metabólico, o equilíbrio da dinâmica emocional do paciente e de sua família e a monitorização contínua dos potenciais riscos de complicações agudas e crônicas. Ao longo do tempo, apesar das novas preparações de insulinas, mais de $50 \%$ dos pacientes evoluem com complicações microvasculares. É importante salientar que o controle glicêmico inadequado nos primeiros cinco anos de evolução do diabetes parece exercer papel importante na gênese das complicações macrovasculares (40). O ensaio clínico DCCT demonstrou claramente que o tratamento intensivo com insulina, por meio de múltiplas doses diárias ou por meio de bomba de infusão contínua subcutânea de insulina quando comparado ao tratamento convencional, proporcionou redução significante na incidência e na progressão das complicações microvasculares $(73,74)$.
Em conclusão, a preferência pelo tratamento intensivo do diabetes melito do tipo $1 \mathrm{com}$ insulinas mais previsíveis, ou seja, com maior reprodutibilidade de ação e menor variabilidade intra e interindividuais, pelo esquema basal/bólus ou pelas bombas de infusão contínua subcutânea de análogos rápidos de insulinas, objetiva mimetizar a liberação diária fisiológica de insulina pelas células- $\beta$. Estes métodos de prescrição de insulinas promovem importantes reduções das complicações micro e macrovasculares, tanto em crianças como em adultos. Embora, ultimamente, o uso de bombas de infusão contínua subcutânea de insulina venha crescendo, é importante citar que a terapia com múltiplas aplicações diárias de insulinas tem alcançado resultados semelhantes, inclusive em crianças em período pré-escolar (75-83). O uso de bombas para o tratamento do diabetes melito tipo 1 será discutido com detalhes em outro artigo desta edição.

\section{REFERÊNCIAS}

1. Arduino F. O diabetes através dos tempos. In: Arduino F. Diabetes mellitus e suas complicações. $2^{\mathrm{a}}$ ed. Rio de Janeiro: Guanabara Koogan; 1973. p. 1-4.

2. Skyler JS, Raptis S. Symposium on biosyntetic human insulin. Diabetes Care. 1981;4:139-264.

3. Bliss M. The history of insulin. Diabetes Care. 1993;16(I3 Suppl):S4-7.

4. King KM. A history of insulin: from discovery to modern alternatives. Br J Nurs. 2003;12(19):1137-41.

5. Brostoff JM, Keen H, Brostoff J. A diabetic life before and after insulin era. Diabetologia. 2007;50:1351-3.

6. Murnaghan JH, Talalay P. John Jacob Abel and the crystallization of insulin. Persp Biol Med. 1967;10:334-81.

7. Sonksen $\mathrm{PH}$. The evolution of insulin treatment. Clin Endocrinol Metab. 1977;6:481-97.

8. Alberti G. Lessons from the history of insulin. Diabetes Voice. 2001;46(4):33-4.

9. Hirsch IB. Insulin analogues. N Engl J Med. 2005;352:174-83.

10. Wagstaff AJ, Reynolds NA. Insulin aspart: a review of its use in the management of type 1 or 2 diabetes mellitus. Drugs. 2004;64(17):1957-4.

11. Bolli GB, Owens DR. Insulin glargine. Lancet. 2000;356:443-5.

12. Heineman $L$, Linkeschova $R$, Rave $K$. Time-action profile of the long-acting insulin analog insulin glargine (HOE901) in comparison with those of NPH insulin and placebo. Diabetes Care. 2000;23:644-9.

13. Pavlic-Renar I, Prasek M, Djoic M. Insulin detemir- A novel basal insulin. Diabetologia Croat. 2003;32(4):163-7.

14. Robertson KJ, Schoenle E, Gucevt Z, Mordhorst L, Gall MA, Ludvgsson J. Insulin detemir compared with NPH insulin in children and adolescents with type 1 diabetes. Diabet Med. 2007;24:27-34.

15. Home P, Bartley P, Russel-Jones D, Hanaire-Broutin H, Heeg J-E. Insulin detemir offers improved glycemic control compa- 
red with NPH insulin in people with type 1 diabetes. Diabetes Care. 2004;27:1081-7.

16. Guntur VP, Dhand R. Inhaled insulin: extending the horizons of inhalation therapy. Respir Care. 2007;52(7):911-22.

17. Ghosh S, Collier A. Inhaled insulins. Postgrad Med J. 2007;83(977):178-81.

18. Pham DQ, Coher $\mathrm{H}$, Chu V. Inhaled human [rDNA origin] insulin, a novel formulation for diabetes mellitus. J Clin Pharmacol. 2007;47(7):890-903.

19. Eisenbarth GS. Type 1 diabetes mellitus: a chronic autoimmune disease. N Engl J Med. 1986;314(21):1360-8.

20. American Diabetes Association. Report of the Expert Committee on the Diagnosis and Classification of Diabetes Mellitus. Diabetes Care. 1997;20:1183-97.

21. Genuth S. Insights from the diabetes control and complications trial/epidemiology of diabetes interventions and complications study on the use of intensive glycemic treatment to reduce the risk of complications of type 1 diabetes. Endocr Pract. 2006;12(Suppl 1):34-41.

22. Nathan DM, Cleary PA, Backlund JY, Genuth SM, Lachin JM, Orchard TJ, et al. Intensive diabetes treatment and cardiovascular disease in patients with type 1 diabetes. N Engl J Med. 2005;353:2643-53.

23. Eisenbarth GS. Update in type 1 diabetes. J Clin Endocrinol Metab. 2007;92:2403-7.

24. Johnsson S. Retinopathy and nephropaty in diabetes mellitus: comparison of the effects of two forms of treatment. Diabetes. 1960;9:1-8.

25. Tubiana-Rufi N, Coutant R, Bloch J. Special management of insulin lispro in continuous subcutaneous insulin infusion in young diabetic children: a randomized cross-over study. Horm Res. 2004;62:265-71.

26. Holcomb JH, Zalani S, Arora VK, Mast CJ. Comparison of insulin lispro with regular human insulin for the treatment of type 1 diabetes in adolescents. Clin Ther. 2002;24:629-38.

27. Ford-Adams ME, Murphy NP, Moore EJ. Insulin lispro: a potencial role in preventing nocturnal hypoglycaemia in young children with diabetes mellitus. Diabet Med. 2003;20:656-60.

28. Hanas R, Urakami T, Jarosz-Chobot P, Deeb LC, Danne T, Bangstad H-J. ISPAD Clinical Practice Consensus Guidelines 2006-2007. Insulin treatment. Pediatric Diabetes. 2007;8:88-102.

29. Dunger DB, Acerini CL, Edge JA, Ford-Adams M, Ong KK, Murphy NP, et al. Randomized cross-over trial of insulin glargine plus lispro or NPH plus regular human insulin in adolescents with type 1 diabetes on intensive insulin regimens. Diabetes Care. 2003;26:799-804.

30. Porcellati F, Rossetti P, Pampanelli S. Better long-term glycaemic control with the basal insulin glargine as compared with $\mathrm{NPH}$ in patients with type 1 diabetes mellitus given meal-time lispro insulin. Diabet Med. 2004;21:1213-20.

31. Hanas R, Adolfsson P. Insulin pumps in pediatric routine care improve long-term metabolic control without increasing the risk of hypoglycemia. Pediatr Diabetes. 2006;7:25-31.

32. Nahata L. Insulin therapy in pediatric patients with type 1 diabetes: Continuous subcutaneous insulin infusion versus multiple daily injections. Clin Pediatr. 2006;45:503-8.

33. Hirsch IB, Bode BW, Garg S, Lane WS, Sussman A, Hu P. Continuous subcutaneous insulin infusion (CSII) of insulin aspart versus multiple daily injection of insulin aspart/insulin glargine in type 1 diabetic patients previously treated with CSII. Diabetes Care. 2005;28:533-8.

34. Alemzadeh R, Berhe T, Wyatt DT. Flexible insulin therapy with glargine insulin improved glycemic control and reduced seve- re hypoglycemia among preschool-aged children with type 1 diabetes mellitus. Pediatrics. 2005;115:1320-4.

35. Lteif AN, Schwenk WF. Type 1 diabetes mellitus in early childhood. Glycemic control and associated risk of hypoglycemic reactions. Mayo Clin Proc. 1999;74:211-6.

36. Becker DJ, Ryan CM. Hypoglycemia: a complication of diabetes therapy in children. Trends Endocrinol Metab. 2000;11:198-202.

37. Northam EA, Rankins D, Cameron FJ. Therapy insight: the impact of type 1 diabetes on brain development and function. Nat Clin Pract Neurol 2006;2(2):78-86.

38. Dixon B, Chase HP, Burdick J, Fiallo-Scharer R, Walravens $P$, Klingensmith $\mathrm{G}$, et al. Use of insulin glargine in children under age 6 with type 1 diabetes. Pediatric Diabetes. 2005;6:150-4.

39. DCCT Research Group. The relationship of glycemic exposure ( $\mathrm{HbA} 1 \mathrm{c})$ to the risk of development and progression of retinopathy in the diabetes control and complications trial. Diabetes. 1995;44:968-83.

40. The Diabetes Control and Complications Trial/Epidemiology of Diabetes Interventions and Complication (DCCT/EDIC) Study Group. Intensive diabetes treatment and cardiovascular disease in patients with type 1 diabetes. $N$ Engl $J$ Med. 2005;353:2643-53.

41. Deeb LC, Holcombe JH, Brunelle R. Insulin lispro lowers postprandial glucose in prepubertal children with diabetes. Pediatrics. 2001;108:1175-9.

42. Valle D, Santoro D, Bates P. Italian multicentre study of intensive therapy with insulin lispro in 1184 patients with type 1 diabetes. Diabetes Nutr Metab. 2001;14:126-32.

43. Cypryk K, Forlani G, Hadden DR, Lawall H, Lopaschuk GD, Tamas $\mathrm{G}$. Insulin lispro. A review of its use in the management of diabetes mellitus. Drugs. 2007;67(3):407-34.

44. Cherubini V, Lannilli A, Lafusco D, Cardella F, Giamprimi MS, Fanelli C. Premeal insulin treatment during basal-bolus regimen in young children with type 1 diabetes. Diabetes Care. 2006;29(10):2311-2.

45. Becker RH, Frick AD, Burger F, Potgieter JH, Scholtz $\mathrm{H}$. Insulin glulisine, a new rapid-acting insulin analogue, displays a rapid time-action profile in obese non-diabetic subjects. Exp Clin Endocrinol Diabetes. 2005;113(8):435-43.

46. Kamal AD, Bain SC. Insulin glulisine: efficacy and safety compared with other rapid-acting insulin analogues. Expert Opin Drug Saf. 2007;6(1):5-7.

47. Becker RH. Insulin glulisine complementing basal insulins: a review of structure and activity. Diabetes Technol Ther. 2007;9(1):109-21.

48. Rave K, Kein O, Frick AD, Becker RHA. Advantage of premealinjected insulin glulisine compared with regular human insulin in subjects with type 1 diabetes. Diabetes Care. 2006;29:1812-7.

49. Kotanko P, Skrabal F, Bachar M, Kitzler TM. Evaluation of treatment adherence in type 1 diabetes: a novel approach. Eur $\mathrm{J}$ Clin Invest. 2007;37(3):207-13.

50. Oikine R, Bernbaum M, Mooradian AD. A critical appraisal of the role of insulin analogues in the management of diabetes mellitus. Drugs. 2005;65(3):325-40.

51. Pieber TR, Zdravkovic M, Draeger E, Endahl AL, Regittinig W, Gorzer E, et al. A double-blind, randomized, dose-response study investigating the pharmacodynamic and pharmacokinetic properties of the long-acting insulin analog detemir. Diabetes Care. 2005;28(5):1107-12.

52. Chapman TM, Perry CM. Spotlight on insulin detemir in type 1 and 2 diabetes mellitus. Bio Drugs. 2005;19(1):67-9. 
53. Ratner RE, Hirsch IB, Neifing JL, Glarg SK, Mecca TE, Wilson CA. Less hypoglycemia with insulin glargine in intensive insulin therapy for type 1 diabetes. US Study Group of Insulin Glargine in Type 1 Diabetes. Diabetes Care. 2000;23:639-43.

54. Bolli GB. Clinical strategies for controlling peaks and valleys: type 1 diabetes. Int J Clin Pract Suppl. 2002;129:65-74.

55. Hermansen K, Fontaine P, Kukolja KK, Peterkova V, Leth G, Gall $M A$. Insulin analogues (insulin detemir and insulin aspart) versus tradicional human insulins (NPH insulin and regular human insulin) in basal-bolus therapy for patients with type 1 diabetes. Diabetologia. 2004;47:622-9.

56. Mortensen H, Kocova M, Teng LY, Keiding J, Bruckner I, Philotheou A. Biphasic insulin aspart vs. human insulin in adolescents with type 1 diabetes on multiple daily insulin injections. Pediatr Diabetes. 2006;7:4-10.

57. Mortensen HB, Robertson KJ, Anstoot HJ. Insulin management and metabolic control of type 1 diabetes mellitus in childhood and adolescence in 18 countries. Hvidore Study Group on Childhood Diabetes. Diabet Med. 1998;15:752-9.

58. Quattrin T, Belanger A, Bohannon NJV, Schwartz SL. Efficacy and safety on inhaled insulin (Exubera) compared with subcutaneous insulin therapy in patients with type 1 diabetes. Diabetes Care. 2004;27:2622-7.

59. Dunger DB, Acerini, Watts AP, Ong KL, Edge JA, Moore EJ, et al. Insulin lispro: a potencial role in preventing nocturnal hypoglycemia in young children with diabetes mellitus. Diabet Med. 2003;20:656-60.

60. Skyle JS, Weinstock RS, Raskin P. Use of inhaled insulin in a ba$\mathrm{sal} / \mathrm{bolus}$ insulin regimen in type 1 diabetic subjects: a 6-month, randomized, comparative trial. Diabetes Care. 2005;28:1630-5.

61. Ludvigsson J, Hanas R. Continuous subcutaneous glucose monitoring improved metabolic control in pediatric patients with type 1 diabetes: a controlled crossover study. Pediatrics. 2003;111:933-8.

62. Deiss D, Hartmann R, Schmidt J, Kordonouri O. Results of a randomized controlled cross-over trial on the effect of continuous subcutaneous glucose monitoring (CGMS) on glycaemic control in children and adolescents with type 1 diabetes. Exp Clin Endocrinol Diabetes. 2006;114(2):63-7.

63. Lagard WH, Barrow FP, Davenport ML, Kang M, G uess HA, Calikoglu AS. Continuous subcutaneous glucose monitoring in children with type 1 diabetes mellitus. a single-blind, randomized, controlled trial. Pediatr Diabetes. 2006;7(3):159-64.

64. Cohen ND, Shaw JE. Diabetes advances in treatment. Internal Medicine Journal. 2007;37:383-388.

65. Tsai EB, Sherry NA, Palmer JP, Herold KC. The rise and fall of insulin secretion in type 1 diabetes mellitus. Diabetologia. 2006;49:261-70.

66. Garg S, Jovanovic L. Relationship of fasting and hourly blood glucose levels to HbA1 values: safety, accuracy, and improvements in glucose profiles obtained using a 7-day continuous glucose sensor. Diabetes Care. 2006;29(12):2644-9.

67. Hernãez Rodriguez R, Conget Donlo I. Glycemic control and new prospects on insulinotherapy in diabetes mellitus type 1 . Rev Clin Esp. 2005;205(6):290-6.

68. Goran MI, Gower BA. Longitudinal study on pubertal insulin resistance. Diabetes. 2001;50:2444-50.
69. Brandou F, Brun JF, Mercier J. Limited accuracy of surrogates of insulin resistance during puberty in obese and lean children at risk for altered glucoregulation. J Clin Endocrinol. 2005;90:761-7.

70. Amiel SA, Sherwin RS, Simonson DC, Lauritano AA, Tamborlane WV. Impaired insulin action in puberty. A contributing factor to poor glycemic control in adolescents with diabetes. N Engl J Med. 1986,315:215-9.

71. Devendra D, Liu E, Eisenbarth GS. Type 1 diabetes: recent developments. BMJ 2004;328:750-754.

72. EURODIAB ACE Study Group. Variation and trends in incidence of childhood diabetes in Europe. Lancet. 2000;355:873-6.

73. The Diabetes Control and Complications Trial Research Group. The effect of intensive treatment of diabetes on the development and progression of long-term complications in insulin-dependent diabetes mellitus. N Engl J Med. 1993;329(14):977-86.

74. Svensson M, Eriksson JW, Dahlquist G. Early glycemic control, age at onset, and development of microvascular complications in childhood-onset type 1 diabetes: a population-based study in northern Sweden. Diabetes Care. 2004;27:955-62.

75. Pickup J, Mattock M, Kerry S. Glycaemic control with continuous subcutaneous insulin infusion compared with intensive insulin injections in patients with type 1 diabetes: meta-analysis of randomised controlled trials. BMJ. 2002;324:705-11.

76. Hamann A, Matthael S, Rosak, Silvestre L. A randomized clinical trial comparing breakfast, dinner, or bedtime administration of insulin glargine in patients with type 1 diabetes. Diabetes Care. 2003;26:1738-44.

77. Doily EA, Weinzimer SA, Sttefer A, Ahern JAH, Vincent M, Tamborlone W. A randomized, prospective trial comparing the efficacy of continuous subcutaneous insulin infusion with multiple daily injections using insulin glargine. Diabetes Care. 2004;27:1554-58.

78. Roze S, Valentine WJ, Zakrzewska KE, Palmer AJ. Health-economic comparison of continuous subcutaneous infusion with multiple daily injection for treatment of type 1 diabetes in the UK. Diabet Med. 2005;22:1239-45.

79. DiMeglio LA, Pottorff TM, Boyd SR, France L, Fineberg N, Eugster EA. A randomized, controlled study of insulin pump therapy in diabetic preschoolers. J Pediatr. 2004;145:380-4.

80. Tubiana-Rufi N, Coutant R, Bloch J, Munz-Licha G, Delcroix C, Montand-Raguideau, et al. Special management of insulin lispro in continuous subcutaneous insulin infusion in young diabetic children: a randomized cross-over study. Horm Res. 2004;62:265-71.

81. Gin $\mathrm{H}$, Hanaire-Broutin $\mathrm{H}$. Reproducibility and variability in the action of injected insulin. Diabetes Metab. 2005;31:7-13.

82. Holleman F, Gale EA. Nice insulin, pity about the evidence. Diabetologia. 2007;50(9):1783-90.

83. Bloomgarden ZT. Insulin treatment and type 1 diabetes topics. Diabetes Care. 2006;29(4):936-44.

Endereço para correspondência:

Antonio Carlos Pires

Rua Francisco Inácio de Carvalho, 259

15014-090 - São José do Rio Preto, SP

E-mail: fpires@terra.com.br 